\title{
Beneficios de la práctica de actividad física durante la pandemia generada por el Covid-19
}

\section{Benefits of physical activity during Covid-19's pandemic}

\author{
Zonaika Posada-López¹ iD; Carolina Vásquez-López¹ (iD
}

1Universidad Católica Luis Amigó. Medellín - Antioquia, Colombia; zonaika.posadalo@amigo.edu.co; carolina.vasquezlo@amigo.edu.co

Cómo citar: : Posada-López, Z.; Vásquez-López, C. 2022. Beneficios de la práctica de actividad física durante la pandemia generada por el Covid-19. Rev. Digit. Act. Fis. Deport. 8(1):e2185. http://doi.org/10.31910/rdafd.v8.n1.2022.2185

Artículo de acceso abierto publicado por Revista Digital: Actividad Física y Deporte, bajo una licencia Creative Commons CC BY-NC 4.0

Publicación oficial de la Universidad de Ciencias Aplicadas y Ambientales U.D.C.A, Institución de Educación Superior Acreditada de Alta Calidad por el Ministerio de Educación Nacional.

Recibido: septiembre 27 de 2021 Aceptado: noviembre 2 de 2021 Editado por: Álvaro José Gracia Díaz

\section{RESUMEN}

Introducción: El Covid-19 y las medidas para mitigarlo generaron cambios en las dinámicas y los hábitos de vida de las personas y uno de los más afectados fue la práctica de la actividad física y el ejercicio, por lo que el propósito inicial fue determinar si las personas realizaron actividad física en casa y de qué manera lo ejecutaron. Objetivo: Identificar el tipo de ejercicio físico realizado por diferentes poblaciones de la ciudad de Medellín y los beneficios percibidos al practicarlo, durante el confinamiento por el Covid-19. Metodología: Mediante una investigación cuantitativa, descriptiva, no experimental, transversal, se identificaron las prácticas de actividad física de 606 personas, entre adultos jóvenes, adultos, adultos mayores y personas en situación de discapacidad, de Medellín, quienes respondieron una encuesta y al cuestionario IPAQ, de manera virtual. Resultados: El 50,7 \% de las personas ejecutaron actividad física en casa durante el confinamiento, siendo los adultos la población con más actividad (58\%) y, los adultos mayores, quienes realizaron menos AF (26,9 \%); quienes la practicaron expresan que fue efectiva, alcanzando sus objetivos (71,6 \%). Conclusiones:
Los ejercicios funcionales fueron aquellos que predominaron en casa; las personas utilizaron los implementos que tenían, ejercitaron las actividades solos, por iniciativa propia y mantuvieron la práctica de AF, para obtener los beneficios físicos. Pese a los beneficios psicológicos que tiene la AF, los adultos mayores y la población en situación de discapacidad no los tienen en cuenta, al momento de motivarse para realizar AF.

Palabras clave: Covid-19; Actividad física; Ejercicio; Salud mental; Confinamiento.

\section{ABSTRACT}

Introduction: Covid-19 and the measures to mitigate it generated changes in the dynamics and life habits of people: one of the most affected habits was the practice of physical activity and exercise, so the initial purpose was to determine if people performed physical activity at home and in what way this was carried out. Objective: To identify the type of physical exercise carried out by different populations of the city of Medellín and the benefits perceived when doing it, during the mandatory confinement 
due to covid-19. Methodology: We identified the physical activity practices of 606 people, including young adults, adults, older adults and people with disabilities, through a quantitative, descriptive, nonexperimental, cross-sectional research, in the city of Medellín. Participants answered a survey and the IPAQ questionnaire virtually. Results: $50.7 \%$ of the people performed physical activity at home during confinement, with adults being the population with the most activity (58\%) and the elderly who performed less PA (26.9\%); Those who carried it out state that it was effective, reaching its objectives $(71.6 \%)$. Conclusions: Functional exercises were those that predominated at home, people used the implements they had, carried out the activities on their own, under their own initiative and maintained the practice of PA to obtain its physical benefits. Despite the psychological benefits of PA, older adults and the population with disabilities do not take them into account when motivating themselves to do PA.

Keywords: Covid-19; Physical activity; Exercise; Mental health; Confinement.

\section{INTRODUCCIÓN}

El 2019 generó grandes cambios en la humanidad, debido a la aparición de un nuevo virus que presentó un alto índice de contagio y de mortalidad, a nivel mundial. Fue reconocida como Covid-19 o Sar-Cov-2 y definida por la Organización Mundial de la salud (OMS, 2019a), como una enfermedad infecciosa con diversos síntomas, como fiebre, tos seca y cansancio, que pueden derivar en casos graves, en neumonía, disnea, anosmia, cefalea crónica y fallas en los sistemas cardiovascular, renal y hepático, asociados a preexistencias de otras enfermedades, como diabetes, asma, cardiopatías, obesidad, entre otras (Lucas Choez et al. 2020). Adicionalmente, es una enfermedad que ha tenido repercusiones durante el 2020 y 2021 en la economía, en el relacionamiento social, en lo político y en la salud mental, lo cual, la convierte en la pandemia, con mayor impacto en el mundo.

Debido a los daños en la salud física, las medidas tomadas en el mundo incluyen la cuarentena y el aislamiento, tanto en personas contagiadas como en aquellas que aún no tienen la enfermedad, pretendiendo mitigarel rápido contagio. Esto ocasionó, que personas de todas las edades y condiciones socioeconómicas hayan tenido que cambiar sus hábitos alimenticios, de vigilia, de ejercitación física, laborales, recreativos, entre otros. Trasladaron los puestos de trabajo, las aulas de clase, los lugares de ejercitación y las demás actividades cotidianas, al interior de sus hogares; se reconstruyeron las dinámicas familiares y habitacionales, compartiendo mayor tiempo con familiares o agudizando la percepción de soledad. Todo lo anterior, generó cambios emocionales que, en ocasiones, fueron menguados por la práctica de algún ejercicio físico, dirigido por diferentes plataformas virtuales, rutinas enviadas por entrenadores o práctica libre, a partir de recuerdos de rutinas o identificación de ejercicios, a través de la Web.

Ahora bien, tal como mencionan Chi et al. (2021), los eventos estresantes pueden desencadenar problemas de salud mental y durante la pandemia, el insomnio, la ansiedad y la depresión han sido los trastornos más estudiados e identificados en las diferentes poblaciones. A éstos, se suman el trastorno de estrés postraumático, las fobias y los trastornos, para el control de los impulsos (Ballena et al. 2021).

Con relación a los grupos etarios, Ballena et al. (2021) mencionan que los niños, al estar en casa encerrados, generaron sentimiento de insatisfacción, al no realizar sus actividades cotidianas; los adolescentes y adultos, se pueden sentir frustrados al ver limitadas sus actividades diarias, así como sentirse nostálgicos, aburridos y nerviosos, debido al distanciamiento social y los adultos mayores, también pudieron sentir cambios, que afectaron su bienestar general y la salud mental. Todas estas afectaciones, más que ser producto de la enfermedad, son resultado de las medidas tomadas para mitigar el contagio (distanciamiento social, aislamiento obligatorio).

En el caso de las personas contagiadas por Covid-19, también se han identificado alteraciones en la salud mental, las más frecuentes fueron el miedo, el estrés y la depresión, especialmente, generadas por las dificultades en el tratamiento, el alto índice de mortalidad de los pacientes y el temor a perder la fuente de ingresos económicos (Ballena et al. 2021).

Pese a lo anterior, existen también factores protectores para estos síntomas; son múltiples las investigaciones y teorías, en las cuales, se intenta identificar la relación positiva que existe entre la actividad física o ejercicio físico y la salud mental. Polanco et al. (2021) mencionan que el ejercicio 
físico y su relación con el bienestar psicológico es ampliamente reconocido, especialmente, al identificar la disminución de síntomas de depresión, tras un programa de ejercicios; de acuerdo con estos autores, el combinar el tratamiento farmacológico y un programa de ejercicio físico pareciera tener un efecto superior en la disminución de los síntomas de depresión, por lo que puede ser un coadyuvante en la terapia que se recibe. Adicionalmente, el utilizar ejercicio, en especial, aeróbico y en compañía de otras personas, mejora las relaciones interpersonales y permite adquirir mayores habilidades sociales; asimismo, se generan cambios en la autoestima, la ansiedad y la calidad de vida.

Siguiendo esta línea, Mikkelsen et al. (2017) manifiestan que el ejercicio genera beneficios en los síntomas de la depresión, la ansiedad y el estrés, además, se evidencian efectos sobre las alteraciones neurológicas y neuro-psiquiátricas, así como en el manejo de los trastornos mentales.

Hay que aclarar que la relación entre ejercicio físico y salud mental no solo se ha estudiado en presencia de enfermedad mental, también se habla sobre sus posibilidades como factor protector, previniendo la aparición de dichos trastornos. De acuerdo con Heckmann Maier et al. (2021), se debe estudiar la posibilidad de generar una relación, a través de investigaciones que demuestren que, realizar ejercicio físico con intensidades moderada y alta en los tiempos libres de los trabajadores, tiene influencia positiva en la salud mental, la satisfacción laboral y la salud física, con énfasis en el control de la presión arterial.

De acuerdo con lo anterior, conocer las consecuencias que ha traído el aislamiento es un tema importante en la actualidad; dicho conocimiento permitirá generar estrategias que posibiliten el potenciar los beneficios que trajo el mismo y generar alternativas de solución para las dificultades que se generaron en múltiples tópicos, incluyendo la salud física y mental de las poblaciones. Frente a la práctica de Actividad física (AF) y ejercicio (E), como se mencionó, el aislamiento preventivo llevó a las personas a realizar ejercicio físico en sus casas, inclusive, se ha observado que personas que antes del confinamiento no realizaban ejercicio, al pasar de las semanas y al tener que continuar confinados, comenzaron a dedicar tiempo a esta actividad, a conseguir implementos, que facilitaran la práctica e, incluso, a cambiar hábitos de alimentación, sueño y movimiento, lo cual, es un tema de interés en el ámbito de la actividad física, el ejercicio y el deporte, permitiendo generar alternativas, que permitan mitigar lesiones, orientar adecuadamente la práctica y luchar contra uno de los factores de riesgo para el Covid-19: el sedentarismo y la obesidad.

En Medellín, se aumentaron las ventas de implementos de gimnasio, los influencer fitness empezaron a marcar las pautas de la alimentación y el ejercicio físico, en este tiempo, muchos volvieron el ejercicio un hábito. Por ello, la presente investigación tuvo como objetivos identificar si las personas realizaron actividad física en casa, cuáles beneficios identificaron las poblaciones que lo practicaron, visualizar qué actividades predominaron y si las personas tuvieron orientación y acompañamiento al realizar actividad física o ejercicio.

\section{MATERIALES Y MÉTODOS}

La presente investigación es cuantitativa, no experimental, descriptiva con diseño transversal, debido a que solo se realizó una medición con 2 instrumentos, aplicados simultáneamente.

La población estudiada fueron los habitantes de la ciudad de Medellín, agrupados en 4 poblaciones de interés: jóvenes, adultos, adultos mayores y personas en situación de discapacidad, teniendo en cuenta que cada uno de estos grupos tiene diferencias en cuanto a la práctica de actividad física y ejercicio. Debido a que el universo de estudio es muy amplio, se decidió por la metodología bola de nieve, para la extracción de la muestra.

Con lo anterior, la muestra de la investigación estuvo conformada por 231 jóvenes, 212 adultos, 78 adultos mayores y 85 personas con discapacidad, dentro de las cuales, se encontraban hombres y mujeres, de diferentes estratos socioeconómicos.

Los instrumentos para recolectar la información fueron una encuesta que fue diligenciada de manera virtual y el cuestionario Internacional de Actividad Física (IPAQ, por sus siglas en idioma inglés), que contiene un grupo de 4 cuestionarios; el propósito de este cuestionario es obtener datos internacionalmente comparables, relacionados con actividad física concernientes con la salud; de allí, que haya sido validado en más de 12 países, encontrando propiedades psicométricas, que le permiten ser utilizado en diferentes lugares e idiomas, midiendo prevalencia de participación 
en actividad física (Carrera, 2017). El indicador de actividad física, se expresa en MET (minuto/semana), que son formas de medir el gasto energético, calculando la tasa metabólica basal y los minutos de actividad física.

Para el análisis de la información, se utilizaron medidas estadísticas de tendencia central y dispersión, a través del paquete estadístico SPSS; este permite hacer análisis descriptivo y multivariante, por lo que fue útil para hacer los cálculos de las medidas de tendencia central y de dispersión, así como el cruce de variables.

El protocolo para la medición revisado por el comité de ética de la Universidad Católica Luis Amigó y los participantes dieron su consentimiento, mediante formulario electrónico. La investigación, se rigió bajo la resolución 8430 de 1993 de MinSalud, considerándose, además, como una investigación sin riesgo, de acuerdo con la resolución (Ministerio de Salud, 1993).

Tabla 1. Datos de la muestra.

\section{RESULTADOS Y DISCUSIÓN}

En total participaron 606 sujetos, 263 hombres y 343 mujeres y se distribuyeron en 4 grupos de interés: adultos jóvenes, adultos, adultos mayores y personas en situación de discapacidad, tal como se observa en la tabla 1. Estos grupos, se tuvieron en cuenta, teniendo como referencia la diferencia en los hábitos y necesidades en la práctica de actividad física. Con relación a otros datos sociodemográficos, se encontró que la mayoría de los encuestados vive en estratos socioeconómicos 1, 2 y 3 (89,27\%), siendo predominante en las personas en situación de discapacidad, quienes participaron de la investigación (solo el 1,18\% vivían en estratos 4 al 6).

\begin{tabular}{|l|c|c|c|c|c|}
\hline & $\begin{array}{c}\text { Adulto } \\
\text { joven }\end{array}$ & Adulto & $\begin{array}{c}\text { Adulto } \\
\text { Mayor }\end{array}$ & Discapacidad & Total \\
\hline $\begin{array}{l}\text { Total } \\
\text { encuestados }\end{array}$ & 231 & 212 & 78 & 85 & 606 \\
\hline Hombres & 91 & 91 & 31 & 50 & 263 \\
\hline Mujeres & 140 & 121 & 47 & 35 & 343 \\
\hline
\end{tabular}

De acuerdo con las diferentes necesidades de cada población, se realizaron preguntas relacionadas con la práctica de actividad física antes y durante el confinamiento; en la población adulta joven, el 87,1 \% (202 personas) manifestó que le gusta realizar ejercicio y el 81,9 \% (190), realizaban ejercicio antes del confinamiento, mencionando, dentro de ellos, los ejercicios cardiovasculares ( $25 \%)$, gimnasio $(15,5 \%)$ y otras actividades, tales como caminar, natación, judo, fútbol, baloncesto y ciclismo (44,6\%).

Con relación a los beneficios percibidos tras la práctica de ejercicio, se encontró que los jóvenes perciben que la práctica trae para ellos beneficios físicos $(86,6$ $\%)$ y beneficios psicológicos (76,7 \%); algunos de los físicos descritos fueron: me ayuda a mejorar la salud, incrementa rendimiento, fuerza y coordinación, más energía cuando realizó actividad física, me siento más vital, cambios en el sistema músculo esquelético y en la composición corporal, reducción de tejido graso y fortalecimiento del sistema inmunológico. Por su parte, dentro de los beneficios psicológicos, se encontraron respuestas relacionadas a la liberación del estrés y la ansiedad, ayuda en los patrones de sueño y en la concentración, control de emociones, incremento de la motivación, satisfacción y bienestar general, así como incremento de la autoestima.

De igual manera, durante el confinamiento, 133 adultos jóvenes manifestaron realizar actividad física en casa; algunas actividades reportadas fueron entrenamiento funcional, parkour, break dance, 
baile, rumba aeróbica, zumba, trabajos de fuerza con mancuernas, yoga, estiramientos, entre otros. El $61,65 \%$ realizaron las actividades solos, mientras que el 34,58 \% reportaron estar acompañados. Es importante mencionar que 114 personas expresaron haber comenzado a practicar AF en casa por iniciativa propia, mientras que 19 expresaron que fue por la influencia de alguien más.

Con relación a los materiales para la práctica en casa, la mayoría contaba con implementos, como colchonetas, mancuernas, bandas elásticas, steps, bicicletas estáticas; hubo 13 personas que manifestaron no contar con ningún material y 10 que expresaron utilizar elementos propios del hogar (botellas, palos de escoba, muebles de la casa y morrales), para la práctica. Finalmente, con relación a la experiencia de entrenar en casa, 81 adultos jóvenes $(60,9 \%)$ calificaron la experiencia, como buena; 43, como regular (32,3\%) y 4, como mala $(3,0 \%)$.

En la población de adulto participaron 212 personas, 91 hombres y 121 mujeres, con edades comprendidas entre los 23 y los 60 años de edad; el 85,1 \% (183) manifestó que le gusta realizar ejercicio; el 76,3\% (164) realizaban ejercicio antes del confinamiento, como ejercicios artes marciales o natación 69,5\% (114), asistir al gimnasio 18,9 \% (31) y otros ejercicios cardiovasculares $11,5 \%$ (19).

Al igual que en la población anterior, los resultados obtenidos frente a los beneficios percibidos cuando se practica AF obtuvieron puntajes similares; los adultos expresaron que sí identifican beneficios físicos $(86,0 \%)$ y psicológicos (79,1\%). Los beneficios que describen a nivel físico son tener mejor salud, más resistencia, fuerza, más energía, mejora en la respiración, menos dolor en las piernas, disminución en los dolores de cabeza, disminución en las crisis de asma, pérdida de peso, mejora en los patrones de sueño, menor sensación de cansancio entre otros; a nivel psicológico, las respuestas apuntan a la liberación de estrés, estar activos, concentrados, con más energía, incremento de la autoestima, positivismo, control de emociones, motivación y sensación de bienestar.

Al indagar sobre la práctica de AF en la cuarentena, 123 personas manifestaron realizarla en casa; la mayoría (95), lo hizo por iniciativa propia y solos (73). Con relación a los ejercicios desarrollados, se encontraron estiramientos, fuerza con y sin materiales, ejercicios cardiovasculares, como aeróbicos, baile, simuladores, yoga y ejercicio funcional. Algunos adultos expresaron el uso de dispositivos móviles, plataformas, como YouTube y Apps de ejercicio, así como la conexión virtual con entrenadores, nutricionistas y deportólogos. Al indagar por implementos, expresaron contar con pesas, sogas, colchonetas, bandas, simuladores, caminadoras, balones, conos y consolas de videojuego.

Los adultos mayores, por su parte, constituyeron la muestra más pequeña del estudio; en ella, participaron personas entre los 60 y los 79 años de edad, donde el 56,8 \% (46) manifestó que le gusta realizar ejercicio y el 51,9 \% (42) realizaban ejercicio antes del confinamiento. Los ejercicios que más practicaban eran caminar $(38,5 \%)$, ejercicios cardiovasculares $(8,6 \%)$, la danza y el trote $(5,0 \%)$ e ir al gimnasio (3,7\%).

Con relación a los beneficios que perciben al realizar $A F$, los adultos mayores expresaron que identifican beneficios físicos (62,8\%), pero son menos quienes observan beneficios psicológicos (51,3 \%), en comparación con las poblaciones anteriormente descritas; dentro de los beneficios físicos algunos identifican que se cansan menos en las actividades cotidianas, disminuye dolores, mejora la movilidad y ayuda a la reducción de peso; por su parte, en los beneficios psicológicos, manifiestan sentirse más tranquilos, olvidan los problemas, reduce el estrés y la ansiedad, mejora la concentración y se sienten más felices y animados.

Al identificar quienes practicaron AF en la pandemia, se encontró que 21 adultos mayores continuaron con la práctica; la mayoría de ellos (16), expresa que lo hizo por iniciativa propia, mientras 14 personas realizaban la AF solas y 7 , acompañadas. Con relación a las actividades realizadas en casa manifestaron que caminar, actividades funcionales virtuales, los estiramientos y aeróbicos en casa, fueron los preferidos. Se destaca, además, que muchos adultos mayores recurrieron para estas actividades, al apoyo de familiares, con el uso de plataformas, como YouTube e Instagram y a las conexiones por zoom, especialmente, con los profesores del Inder Medellín $y$, a diferencia de las poblaciones anteriores, la mayoría de encuestados, manifestaron no contar con materiales; algunos tenían colchonetas y utilizaron materiales de casa. 
En la población con discapacidad participaron personas con edades comprendidas entre los 18 y los 79 años de edad, frente a los tipos de discapacidad, como se observa en la tabla 2; la discapacidad que predominó fue la física, seguida por la intelectual, la psicosocial y la sensorial.

Tabla 2. Tipo de discapacidad.

\begin{tabular}{|c|l|c|c|c|}
\hline \multicolumn{2}{|c|}{} & Frecuencia & Porcentaje & Porcentaje acumulado \\
\hline \multirow{4}{*}{ Válidos } & Física & 41 & 48,2 & 48,2 \\
\cline { 2 - 5 } & Intelectual & 19 & 22,4 & 70,6 \\
\cline { 2 - 5 } & Otra & 1 & 1,2 & 71,8 \\
\cline { 2 - 5 } & Psicosocial & 13 & 15,3 & 87,1 \\
\cline { 2 - 5 } & Sensorial & 11 & 12,9 & 100,0 \\
\cline { 2 - 5 } & Total & 85 & 100,0 & \\
\hline
\end{tabular}

Esta población manifestó ser activa físicamente; 65 personas expresaron que les gustaba realizar AF y 62 $(72,9 \%)$, practicar ejercicio antes de la pandemia. Dependiendo de la discapacidad, se encontró que muchas de las actividades reportadas incluían deportes adaptados (Boccia, goalball, powerlifting) y otras actividades, tales como nadar, caminar, ir al gimnasio y montar a caballo.

Ahora bien, al igual que en la población anterior, identifican más beneficios físicos (62,4 \%) que psicológicos (45,9\%), a la hora de realizar AF. En los físicos, se nombran mejor rendimiento físico, mayor flexibilidad, resistencia, fuerza muscular, mejora en la respiración, disminución de peso, mejora en la postura, más energía y alguien manifiesta que le ha servido de factor protector contra el Covid-19; con relación a los beneficios psicológicos manifiestan el tener autoconfianza, tranquilidad, fortaleza mental, mejora en las relaciones sociales, disminución en la ansiedad y el estrés, confianza en sí mismo, mejora en el estado de ánimo y la actitud.
En el confinamiento, 30 personas en situación de discapacidad expresaron continuar con la práctica de AF en casa; de ellos, 22 dijeron realizarlo por iniciativa propia y la mitad, en compañía de alguien más. Las actividades más reportadas fueron ejercicios funcionales, rumba, abdominales, clases virtuales diversas y estiramientos; para la realización de estos ejercicios, muchas personas contaban con profesores y entrenadores virtuales $y$, otros, se apoyaron en familiares para la práctica. Los implementos que reportaron tener fueron pesas, bandas, barras, discos, colchonetas, botellas, lazos y elípticas.

Al preguntar sobre cuáles eran los motivos para realizar AF en los días de cuarentena, las respuestas fueron: mantener el estado físico, estar ocupado y seguir en forma, para alcanzar las metas deportivas.

Con el IPAQ, se calculó la frecuencia de práctica de actividad física en casa durante el confinamiento, de acuerdo con los minutos de actividad; los mismos, permitieron expresar dicha actividad en alta, moderada y baja, de acuerdo con los criterios que se describen en la tabla 3.

Tabla 3. Clasificación de los niveles de actividad física de acuerdo con IPAQ.

\begin{tabular}{|c|c|}
\hline Nivel de actividad física & Descriptor \\
\hline \multirow[t]{2}{*}{ ALTO } & $\begin{array}{l}7 \text { días a la semana, en los que se realizan caminatas o actividades de } \\
\text { moderada o alta intensidad, logrando, mínimo } 3.000 \text { MET } \\
\text { min/semana }\end{array}$ \\
\hline & $\begin{array}{l}\text { Cuando se reporta actividad vigorosa, al menos durante } 3 \text { días a la } \\
\text { semana, que alcanza } 1.500 \mathrm{MET} \mathrm{min} / \mathrm{semana}\end{array}$ \\
\hline \multirow[b]{2}{*}{ MODERADO } & 3 o más días de actividad vigorosa, por al menos 20 minutos diarios \\
\hline & $\begin{array}{l}5 \text { o más días de actividad moderada o caminata, por al menos } 30 \\
\text { minutos diarios }\end{array}$ \\
\hline \multirow{2}{*}{ BAJO } & $\begin{array}{l}5 \text { o más días de combinación de caminata y actividades moderadas } \\
\text { o vigorosas, logrando al menos } 600 \mathrm{MET} \text { min/semana }\end{array}$ \\
\hline & $\begin{array}{l}\text { Cuando el nivel de actividad física no alcanza los niveles } \\
\text { anteriormente descritos }\end{array}$ \\
\hline
\end{tabular}


Los resultados del IPAQ permitieron identificar los hábitos de actividad física de las diferentes poblaciones durante el aislamiento por el Covid-19 y las medidas de confinamiento en casa: el 63,3\% de la población en situación de discapacidad que realizó actividad física, lo hizo de alta intensidad, mientras que el 23,3 \%, de moderada intensidad; en la población adulta joven, el 75,9 \% realizó actividad física de alta intensidad y el 24,1\%, de intensidad baja y moderada. Por su parte, en la población adulta que realizó actividad física durante el confinamiento, el 69,9 \% lo hizo de lata intensidad, el 19,5 de moderada y el 10,6 \% de baja intensidad; valores similares obtuvieron los adultos mayores, identificando que el 42,9\% realizó la actividad física con alta intensidad, el 33,3\% de moderada y el 23,9 $\%$ de baja intensidad; se resalta, además, que el grupo de adulto mayor fue el que menos actividad física realizó, teniendo en cuenta los METs, tal como se observa en la tabla 4.

Tabla 4. Estadísticos IPAQ por población.

\begin{tabular}{|l|c|c|c|c|}
\hline & $\begin{array}{c}\text { Adulto } \\
\text { joven }\end{array}$ & Adulto & Adulto mayor & Discapacidad \\
\hline Media & 4611,15 & 4633,56 & 2219 & 4061,87 \\
\hline $\begin{array}{l}\text { Desviación } \\
\text { típica }\end{array}$ & 5143,78 & 5806,13 & 2149,31 & 4131,78 \\
\hline Mínimo & 16,5 & 113 & 352 & 180 \\
\hline Máximo & 28860 & 40770 & 6974 & 20400 \\
\hline Cuenta & 133 & 123 & 21 & 30 \\
\hline
\end{tabular}

De acuerdo a lo anterior, el 50,7 \% de la población encuestada realizó actividad física en casa durante el confinamiento por Covid-19, con relación al acompañamiento que tuvieron; los resultados dependieron de la población, tal como se observa en la tabla 5.

Tabla 5. Recibió orientación o acompañamiento cuando realizó AF durante el confinamiento.

\begin{tabular}{|l|c|c|c|c|c|}
\hline & Adulto joven & Adulto & Adulto mayor & Discapacidad & Total \\
\hline Si & 53 & 51 & 8 & 22 & 134 \\
\hline No & 75 & 65 & 13 & 8 & 164 \\
\hline
\end{tabular}

Es importante aclarar que no en todos los casos la orientación fue brindada por un profesional, lo cual, fue especialmente evidente en las poblaciones de adulto mayor y discapacidad, en donde el principal apoyo fueron familiares.
Con relación a la efectividad del entrenamiento en casa, tal como se observa en la tabla 6 , el 71,6 \% de las personas encuestadas manifestaron que las actividades realizadas fueron efectivas, de acuerdo con los objetivos que se habían propuesto. 
Tabla 6. Efectividad del entrenamiento en casa.

\begin{tabular}{|l|c|c|c|c|c|c|c|c|c|c|}
\hline \multirow{2}{*}{} & \multicolumn{2}{|c|}{ Adulto joven } & \multicolumn{2}{|c|}{ Adulto } & \multicolumn{2}{|c|}{ Adulto Mayor } & \multicolumn{2}{c|}{ Discapacidad } & \multicolumn{2}{|c|}{ Total } \\
\cline { 2 - 12 } & ni & $\%$ & ni & $\%$ & ni & $\%$ & ni & $\%$ & ni & $\%$ \\
\hline 0 & 2 & 1,5 & 2 & 1,6 & 1 & 4,7 & 0 & 0 & 5 & 1,6 \\
\hline 1 & 0 & 0 & 0 & 0 & 1 & 4,7 & 0 & 0 & 1 & 0,3 \\
\hline 2 & 1 & 0,7 & 0 & 0 & 0 & 0 & 1 & 3,3 & 2 & 0,6 \\
\hline 3 & 0 & 0 & 1 & 0,8 & 0 & 0 & 0 & 0 & 1 & 0,3 \\
\hline 4 & 0 & 0 & 1 & 0,8 & 1 & 4,7 & 0 & 0 & 2 & 0,6 \\
\hline 5 & 10 & 7,5 & 10 & 8,1 & 0 & 0 & 4 & 13,3 & 24 & 7,8 \\
\hline 6 & 9 & 6,7 & 12 & 9,7 & 1 & 4,7 & 0 & 0 & 22 & 7,1 \\
\hline 7 & 9 & 6,7 & 21 & 17,0 & 0 & 0 & 0 & 0 & 30 & 9,7 \\
\hline 8 & 41 & 30,8 & 35 & 28,4 & 6 & 28,6 & 12 & 40 & 94 & 30,6 \\
\hline 9 & 14 & 10,5 & 8 & 6,5 & 1 & 4,7 & 1 & 3,3 & 24 & 7,8 \\
\hline 10 & 47 & 35,3 & 33 & 26,8 & 10 & 47,6 & 12 & 40 & 102 & 33,2 \\
\hline
\end{tabular}

Una de las repercusiones identificadas del Covid-19 y de las medidas tomadas para mitigar el contagio han sido las afectaciones a la salud mental de la población, tanto en personas contagiadas como en aquellas que no han sufrido la enfermedad (Martínez-Taboas, 2020; Ramírez-Ortiz et al. 2020; Ribot Reyes et al. 2020; Huarcaya-Victoria, 2020; Hernández Rodríguez, 2020); una de las definiciones más referidas del concepto de salud mental es "un estado de bienestar en el cual el individuo es consciente de sus propias capacidades, puede afrontar las tensiones normales de la vida, puede trabajar de forma productiva y fructífera y es capaz de hacer una contribución a su comunidad" (OMS, 2004); asimismo, la OMS (2019b) manifiesta que "la salud mental está relacionada con el bienestar mental y psicológico".

En el enfoque conductual de la salud mental, la forma como las personas se comportan es un factor importante en el proceso de salud-enfermedad, por lo que los hábitos, las conductas "adecuadas" y el estilo de vida, se relacionarían directamente con estar "sano" y el trastorno mental implicaría el "desajuste del hábito" (Restrepo O. \& Jaramillo E. 2012). Desde este enfoque, además, se entiende que el ambiente y el moldeamiento de la conducta están implicados dentro de la salud mental (Macaya et al. 2018); asimismo, desde el enfoque socioeconómico, se consideran las leyes que determinan y regulan los comportamientos normales, la política, la economía, el estilo de vida, el acceso a servicios y productos, así como la capacidad de acceder a procesos de intervención de dicha salud mental, la cual, debe ser considerada un problema público (Macaya et al. 2018).

En el país, se tienen normas que, de acuerdo con lo mencionado por Rojas-Bernal et al. (2018), no responden a las necesidades de salud mental de la población; ejemplo de ello, es la ley 100 de 1993, que limitó la atención psicoterapéutica; la ley 1616 de 2013 intentó sanear la situación, decretando la salud mental, como un derecho de la población y como un tema prioritario en salud pública; sin embargo, y aunque en el plan decenal de salud pública 20122021 la salud mental es una prioridad, el acceso a los diferentes servicios continúa siendo limitado, por lo que, en general, para atención integral en salud mental, los colombianos deben recurrir a métodos legales, situación que se agudizó con el Covid-19 y puso en entredicho, los procesos que promueven la salud mental de la población.

Por ello, alternativas que promueven y protegen la salud mental, tales como la práctica de actividad física y el ejercicio, cobran relevancia en la ciudad. En la investigación realizada, se indagó sobre los efectos que la pandemia y el confinamiento generaron en 
las personas encuestadas, 156 adultos jóvenes, 143 adultos, 46 adultos mayores y 27 personas en situación de discapacidad y manifestaron que evidenciaron cambios en su estado emocional durante la pandemia; dentro de las respuestas más frecuentes, se encontraron estrés, tristeza, miedo, ansiedad, nostalgia, angustia, irritabilidad, aburrición, depresión y temor a enfermar, pero se destaca, también, que no todas las personas que respondieron afirmativamente a dichos cambios emocionales recurrieron a la práctica de $A F$, como alternativa en los meses de confinamiento.

Ahora bien, según la OMS (2021), los niveles de inactividad se incrementan en los países en vías de desarrollo y algunos factores inciden en ello, tales como la pobreza, el crimen, el tráfico, la contaminación ambiental y la poca facilidad recreativa; además, afirma que los países con ingresos bajos tienden poco a la prevención de enfermedades no transmisibles, tales como el sedentarismo. Evidenciando esta hipótesis en el país, en donde muchas personas que participaban previamente en actividades físicorecreativas, continuaron vinculadas a ellas, por medio de la virtualidad; sin embargo, aquellas que no realizaban AF antes de la pandemia, no empezaron, en su mayoría, nuevas actividades.

Esto se evidenció, especialmente, en poblaciones, como las personas adultas mayores y personas en situación de discapacidad; en estas últimas, se pueden observar niveles de salud por debajo de la "normal"; por ejemplo, se encuentran personas con retardo mental, autismo, parálisis cerebral y deficientes visuales, con niveles de salud física, por debajo de aquella que tienen sus pares sin discapacidad; esto, se debe a la falta de programas que estimulan la práctica de actividad física (González Hernández et al. 2012). La inactividad provoca que las personas con discapacidad muestran niveles de obesidad elevados y que aumenten los factores de riesgo coronario, además, se evidencia el uso inapropiado del tiempo libre en diferentes grupos de la sociedad, que incrementa el sedentarismo (Sherrill, 1998).

Los nuevos paradigmas en temas de salud incluyen a la actividad física como factor determinante en la prevención, encontrándose beneficios fisiológicos, sociales y psicológicos, prolongando, además, la calidad de vida (Paffenbarger et al. 1993); por ello, es necesario e imperante que poblaciones vulnerables, especialmente, las personas en situación de discapacidad, practiquen AF con regularidad, convirtiéndose en un hábito. Los resultados de la investigación muestran que la actividad física en la población del área metropolitana no es parte del estilo de vida de las personas en diferentes grupos poblacionales $y$, por ello, no fue practicada por un porcentaje mayor durante el confinamiento, pese a las posibilidades que se tenían, los materiales e, inclusive, el malestar psicológico experimentado, por lo que se requieren programas que incentiven dicha práctica. Lo encontrado fue similar a los hallazgos de Qin et al. (2020), quienes identificaron que el $60 \%$ de los adultos mayores no cumplieron con los requerimientos mínimos de AF, de la OMS.

\section{CONCLUSIONES}

La investigación tuvo varios objetivos; el primero de ellos fue identificar si las personas realizaron AF y ejercicio durante el confinamiento, el cual, se cumplió, identificando que el 50,7 \% de las personas encuestadas realizaron esta actividad, siendo menos personas de las esperadas; la población que más realizó $A F$ en casa fueron los adultos (58 \%), mientras que la población que menos realizó fueron los adultos mayores (26,9\%).

El segundo objetivo consistió en identificar los motivos que los llevaron a realizar AF y E; se identificó, que predominó la iniciativa propia, las personas realizaron las actividades solas, utilizaron plataformas digitales, especialmente, YouTube y clases mediante Zoom, además de entrenadores personalizados y familiares desde la virtualidad, para orientar los ejercicios y aunque se observó que la mayoría tenía en casa implementación deportiva, algunos reportaron ser creativos, a la hora de realizar los ejercicios.

Con relación a los beneficios percibidos a la hora de hacer AF predominaron aquellos relacionados a los elementos físicos, especialmente, referidos a la pérdida o mantenimiento del peso, la resistencia física, la fuerza, la vitalidad, la disminución de dolores y el sentirse con mayor energía, para enfrentar las actividades cotidianas.

Llama la atención que, aunque la mayoría reportó que la pandemia y el confinamiento generaron cambios, a nivel emocional, pocas personas reportaron que la AF les ayudaba a mitigar las emociones negativas experimentadas. 
Agradecimientos. Se agradece a la Universidad Católica Luis Amigó y a todas las personas que participaron de la encuesta. Conflicto de intereses: El manuscrito fue elaborado y revisado por todos los autores, que declaran la ausencia de cualquier conflicto que puede poner la validez de los resultados en riesgo. Todos han contribuido a la investigación presentando los datos reales, que se encontraron al recolectar los datos. Financiación: Este trabajo de investigación fue financiado por la Universidad Católica Luis Amigó y hace parte del grupo de investigación Familia, Desarrollo y Calidad de vida de la Facultad de Psicología y Ciencias Sociales.

\section{REFERENCIAS}

1. BALLENA, C.L.; CABREJOS, L.; ÁVILA, Y.; GONZÁLEZ, C.G., MEJÍA, G.E.; RAMOS, V.; BARBOZA, J.J. 2021. Impacto del confinamiento por COVID-19 en la calidad de vida y salud mental. Revista del Cuerpo Médico Hospital Nacional Almanzor Aguinaga Asenjo. 14(1):87-89. https://doi.org/10.35434/rcmhnaaa.2021.141.904

2. CARRERA, Y. 2017. Cuestionario internacional de actividad física (IPAQ). Revista enfermería del trabajo. 7(11):49-54.

3. CHI, X.; LIANG, K.; CHEN, S.T.; HUANG, Q.; HUANG, L.; YU, Q.; TIANYOU, G.; STUBSS, B.; MAHBUD AHOSSAIN, M.; YEUNG, ALBERT, Y.; ZHAOWEI, K.; ZOU, L. 2021. Mental health problems among Chinese adolescents during the COVID-19: The importance of nutrition and physical activity. International Journal of Clinical and Health Psychology. 21(3):100218. https://doi.org/10.1016/j.ijchp.2020.100218

4. GONZÁLEZ HERNÁNDEZ, J.; BAÑOS AUDIJE, L.M. 2012. Estudio sobre el cambio de actitudes hacia la discapacidad en clases de actividad física. Cuadernos de Psicología del Deporte. 12(2):101-108.

5. HECKMANN MAIER, B.; SANDOVAL HERMINDA, F.; ARROYO-JOFRÉ, P. 2021. El ejercicio físico como factor protector de estrés en profesores de educación primaria y secundaria: revisión sistemática. Revista Horizonte Ciencias de la Actividad Física. 12(1):70-89.
6. HERNÁNDEZ RODRÍGUEZ, J. 2020. Impacto de la COVID-19 sobre la salud mental de las personas. Medicentro Electrónica. 24(3):578594.

https://doi.org/10.21615/cesmedicina.32.2.6

7. HUARCAYA-VICTORIA, J. 2020. Consideraciones sobre la salud mental en la pandemia de COVID-19. Revista peruana de medicina experimental y salud pública. 37(2):327-334. https://doi.org/10.17843/rpmesp.2020.372.5419

8. LUCAS CHOEZ, M.M.; ZAMBRANO CERÓN, D.G.; GARCÍA ZAMBRANO, C.J.; PINCAY PIN, V.E. 2020. Riesgos y consecuencias de los pacientes contagiados con COVID 19. RECIMUNDO. 4(2):217-225.

https://doi.org/10.26820/recimundo/4. (2). mayo.2020.217-225

9. MACAYASANDOVAL,X.C.; PIHANVYHMEISTER, R.; PARADA, B.V. 2018. Evolución del constructo de salud mental desde lo multidisciplinario. Humanidades Médicas. 18(2):338-355.

10. MARTÍNEZ-TABOAS, A. 2020. Pandemias, COVID-19 y Salud Mental: ¿Qué Sabemos Actualmente? Revista Caribeña de Psicología. 4(2):143-152.

https://doi.org/10.37226/rcp.v4i2.4907

11. MIKKELSEN, K.; STOJANOVSKA, L.; POLENAKOVIC, M.; BOSEVSKI, M.; APOSTOLOPOULOS, V. 2017. Exercise and mental health. Maturitas, 106:48-56.

https://doi.org/10.1016/j.maturitas.2017.09.003

12. MINISTERIO DE SALUD. 1993. Resolución número 8430 de 1993: normas científicas, técnicas y administrativas para la investigación en salud. Disponible desde Internet en: https://www.minsalud.gov.co/sites/rid/Lists/ BibliotecaDigital/RIDE/DE/DIJ/RESOLUCION8430-DE-1993.PDF (con acceso 11/6/2021).

13. ORGANIZACIÓN MUNDIAL DE LA SALUD, OMS. 2004. Promoción de la salud mental: Conceptos, evidencia emergente y práctica. Informe compendiado. Ginebra: OMS.

14. ORGANIZACIÓN MUNDIAL DE LA SALUD, OMS. 2019a. Coronavirus causante del síndrome 


\section{Revista Digital: Actividad Física y Deporte}

respiratorio de Oriente Medio (MERSCoV). Disponible desde Internet en: https:// www.who.int/es/news-room/fact-sheets/ detail/middle-east-respiratory-syndromecoronavirus-(mers-cov) (con acceso el 25/5/2021).

15. ORGANIZACIÓN MUNDIAL DE LA SALUD, OMS. 2019b. Salud mental. Disponible desde Internet en: https://www.who.int/es/newsroom/facts-in-pictures/detail/mental-health (con acceso el 25/5/2021).

16. ORGANIZACIÓN MUNDIAL DE LA SALUD, OMS. 2021. Enfermedades no trasmisibles. Disponible desde Internet en: https://www. who.int/es/news-room/fact-sheets/detail/ noncommunicable-diseases (con acceso el 25/5/2021).

17. PAFFENBARGER, JR. R.S.; HYDE, R.T.; WING, A.L.; LEE, I.M.; JUNG, D.L.; KAMPERT, J.B. 1993. The association of changes in physical-activity level and other lifestyle characteristics with mortality among men. New England journal of medicine. 328(8):538-545.

https://doi.org/10.1056/nejm199302253280804

18. POLANCO, K.M.; LÓPEZ-WALLE, J.M.; MUÑOZNOGUERA, B.C.; VERGARA-TORRES, A.P.; TRISTÁN, J.; ARANGO-DÁVILA, C.A. 2021. Efecto del ejercicio físico sobre los síntomas depresivos en pacientes hospitalizados con depresión. Retos. 43:53-61.

https://doi.org/10.47197/retos.v43i0.88339
19. QIN, F.; SONG, Y.; NASSIS, G.P.; ZHAO, L.; CUI, S.; LAI, L.; WU, Z.; XU, M.; QU, C.; DONG, Y.; WANG, Z.; GEN, X.; ZHAO, C.; FENG, Y.; HAN, Z.; FAN, Z.; ZHAO, J. 2020. prevalence of insufficient physical activity, sedentary screen time and emotional well-being during the early days of the 2019 novel coronavirus (Covid-19) outbreak in China: a national cross-sectional study. The Lancet. Preprint.

20. RAMÍREZ-ORTIZ, J.; CASTRO-QUINTERO, D.; LERMA-CÓRDOBA, C.; YELA-CEBALLOS, F.; ESCOBAR-CÓRDOBA, F. 2020. Consecuencias de la pandemia COVID-19 en la Salud Mental asociadas al aislamiento social. Revista Colombiana de Anestesiología. 48(4):e930. https://doi.org/10.5554/22562087.e930

21. RESTREPO O., D.A.; JARAMILLO E., J.C. 2012. Concepciones de salud mental en el campo de la salud pública. Rev Fac. Nac. Salud Publica. 30 (2).

22. RIBOT REYES, V.D.; CHANG PAREDES, N.; GONZÁLEZ CASTILLO, A.L. 2020. Efectos de la COVID-19 en la salud mental de la población. Revista Habanera de Ciencias Médicas. 19(supl.1).

23. ROJAS-BERNAL, L.Á.; CASTAÑO-PÉREZ, G.A.; RESTREPO-BERNAL, D.P. 2018. Salud mental en Colombia. Un análisis crítico. CES Medicina, 32(2):129-140.

24. SHERRILL, C. 1998. Adapted physical activity, recreation and sport: Crossdisciplinary and lifespan. McGraw Hill. 706p. 